\title{
LA TEORÍA DE LAS DISTINCIONES EN LA EDAD MEDIA Y SU INFLUJO EN LA EDAD MODERNA'
}

\author{
Mauricio Beuchot \\ Instituto de Investigaciones Filológicas. UNAM
}

\section{INTRODUCCIÓN}

Dentro de las propiedades trascendentales del ente, los escolásticos ponían la unidad. Esta unidad consiste en la división externa del ente, esto es, la separación de una cosa con respecto de otra. Fundada en la unidad aparecía la identidad, que era la indivisión interna, la coincidencia de algo consigo mismo. Ya Aristóteles decía que había tres términos relacionados: la identidad, la igualdad y la semejanza. La identidad se da entre substancias, la igualdad entre cantidades y la semejanza entre cualidades -es decir, se aplican a distintas categorías, las tres primeras de la tabla categorial aristotélica. ${ }^{2}$ Opuesta a la identidad (pero no a la unidad) es la distinción. La distinción dice pluralidad, puede excluir la división externa, o de una cosas con respecto de otra, admitiendo sólo la división interna, pero de modo relativo, Es decir, no siempre implica separación; puede bastar, para que haya distinción, otro tipo de división.

Dado que es un concepto transcendental (i.e., en el sentido escolástico de universalísimo), la distinción no tiene propiamente definición; pero puede tener descripción, esto es, puede describirse de modo negativo como la carencia de identidad entre muchos, o la entidad no idéntica de muchos (carentia identitatis inter plura, vel non eadem plurium entitas), 0 también como la negación de la identidad, o la negabilidad de una cosa con respecto de otra (negatio identitatis, vel negabilitas unius de alio in recto vel capacitas verificandi propositionem negativam unius de alio), pues se puede expresar por una proposición negativa: «esto no es aquello» («hoc non est illud»).

1 Agradezco la lectura crítica de este artículo a José Antonio Robles.

2 Cfr. Aristóteles, Metaphysica, V, 11. 
Consideraremos en seguida las diferentes teorías de las distinciones que se presentaron en las escuelas tomista, escotista, ockhamista y suareciana. La tomista y la escotista eran realistas (denominadas «reales»), la ockhamista era nominalista (la llamada «secta de los nominales») y la suareciana trató de sintetizar a todos. Precisamente fue esta última la que más influyó en los modernos. Pero las demás se hicieron presentes en los modernos a través de ella. En algunos casos, los modernos siguieron a los tomistas (tal vez en muy pocos casos); más frecuente fue seguir a los escotistas, a través de los nominalistas y los suarecianos. Los nominalistas tuvieron sin lugar a dudas, mucho influjo, no necesariamente por la mediación de los suarecianos. Veamos primero lo que cada escuela dice de las distinciones, y después tratemos de rastrear cómo influyeron en los modernos.

\section{LAS DISTINCIONES EN LA ESCUELA TOMISTA}

Para Santo Tomás, la distinción es la ausencia (y lo opuesto) de la identidad entre varias cosas o entidades. Dividió la distinción en dos grandes grupos: distinciones reales, que son las que se dan independientemente de la intervención del intelecto o razón; y distinciones de razón, que sólo son producto de la inteligencia. ${ }^{3} \mathrm{La}$ distinción real puede darse por el lado de la forma o por el de la materia y la cantidad. La que se da por la forma es llamada «formal» o «específica», es la que se da en el seno de un género, diferencia específica que lo divide en varias especies. Pues bien, esta distinción formal es más perfecta que la numérica y la funda. La numérica se da, ya en concreto, por virtud de la materia determinada por la cantidad, ya que tal es el principio de individuación y la división numérica se da ente individuos (dentro de una especie, por eso se relaciona con la específica). ${ }^{4}$ Entre los distintos hay oposición de contradicción. ${ }^{5}$ Además, el propio Aquinate habla de que la distinción de razón puede tener dos clases: (a) con fundamento en la cosa y (b) sin fundamento en ella, sino sólo en la mente. Todo esto se ve resumido en el siguiente pasaje: «En las cosas que se significan por los nombres se encuentra una triple diversidad. En efecto, algunas hay que según el ser completo están fuera del alma; y tales son entes completos, como el hombre y la piedra. Pero hay otras que no tienen nada fuera del alma, como los sueños y la imaginación quimérica. Y otras hay que tienen fundamento en la cosa fuera del alma pero el complemento de razón en ellas, en cuanto a lo que es formal, es por la operación del alma, como resulta patente en el universal». ${ }^{6}$

Posteriormente, en la escuela tomista, la distinción real recibió otras divisiones. Se hablaba de una distinción mayor (a la que también se daban los nombres de entitativa, realis simpliciter y realis-realis y de una distinción menor (que también se pasó a llamar modal,

\footnotetext{
3 Cfr. Sto. Tomás, In I Sententiarum, d. 2, q. 5; d. 31, q. 1, a. 2, c.

4 Cfr. Idem, Summa Theologiae, I, q. 47, a. 2, c.; Contra Gentiles, lib. 2, c. 39.

5 Cfr. Contra Gentiles, lib. 4, c. 14.

6 Idem, In I Sententiarum, d. 19, q. 5, a. 1.
} 
y que fue la que quiso después rescatar Suárez), e incluso se llegó a añadir una distinción todavía más débil, rayana en la de razón, a la que se llamó virtual, y se usaba sobre todo para explicar distinciones en Dios; ${ }^{7}$ pero algunos la vieron no tanto como una distinción, sino tan sólo como el fundamento de una distinción; ${ }^{8}$ o incluso se colocaba en las de razón. Asimismo, se daba otra división de la distinción real en adecuada e inadecuada, otra en física y metafísica, y otra en positiva y negativa.

La distinción real mayor es aquella en la que los distintos están separados, como cosas y cosa (res a re), como la que se de entre un hombre y otro o entre el hombre y su ropa; 0 , si están compuestos, son separables de ese modo (ut res a re), al menos por la intervención divina. Y esto, ya sean substancias, como Dios y el mundo, el cuerpo y el alma; o substancias y accidentes, como el hombre y su blancura; o accidentes, como el color y el sabor de una manzana. La distinción real menor o modal es la que se da entre una cosa y sus modificaciones o modos, pero sin que la modificación pueda existir separada, p. ej. en la línea curva se distinguirían las curvatura y la línea; o entre dos modos, ${ }^{9}$ por ejemplo, entre la curvatura y la longitud de una línea. Es la que después resaltará Suárez.

Por otra parte, la distinción adecuada es la que se da entre las cosas que no son idénticas porque una no es parte de la otra, como entre dos individuos, o entre la materia y la forma. La inadecuada se da entre aquellas de las que una es parte de la otra, p. ej. entre cabeza y el resto del cuerpo. Como se ve, son más bien otros nombres para la distinción real mayor y la menor. Lo mismo sucede con estas otras que registran los manuales: (i) física, la que se da entre partes separadas o separables como cosa-y-cosa, y (ii) metafísica, la que se da entre principios o constitutivos, o entre realidades objetivas de una y la misma cosa, por ejemplo entre esencia y existencia; además, (iii) distinción positiva, la que se da entre cosas que pertenecen al orden real, y (iv) negativa, la que se entre una cosa real y otra no-real, o entre dos cosas no-reales, mediante las cuales se aprehende algo real, p. ej. entre el vacío y la nada.

La distinción real tiene como signos : (i) el que una cosa sea origen o principio de la otra, pues se oponen de manera relativa, y esta oposición relativa basta para que haya distinción real; (ii) o a veces se da una separación en acto; (iii) o por lo menos una separabilidad real; (iv) o una composición real, partes reales; (v) y una oposición real, pues las cosas que se oponen realmente implican que una cosa no se identifica realmente con la otra.

La distinción de razón, efectuada por la consideración de la mente, o la que se da en las cosas en cuanto conocidas por un concepto inadecuado y precisivo (i. e., abstractivo), era dividida siguiendo los dos tipos que dejó asentados Santo Tomás: una fundada en la cosa y otra sólo en la razón o la mente. La que es puramente mental fue después llamada «de razón raciocinante», y se da cuando una misma cosa se expresa con varios conceptos, pero que

7 Cfr. J. Martínez de Prado, Controversiae metaphysicales sacrae theologiae ministrae, Compluti: Maria Fernandez, viuda, Universitatis Typographa, 1649, pp. 163 ss.

8 Cfr. A. Goudin, Metaphysica, Lugduni: Antonio Iulieron, 1691, disp. 1, q. 3, a. 2, n. 1.

9 Cfr. F. de Araújo, Commentariorum in universam Aristotelis Metaphysicam tomus secundus, Salmanticae: A. Ramírez, 1631, lib. IX, q. 1, a. 1. Ver además M. Beuchot, Metafísica. La ontología aristotélico-tomista de Francisco de Araújo, México: UNAM, 1987. 
no son diversos en realidad, p. ej. la definición y lo definido (i.e., como «hombre» y «animal racional») y generalmente es la distinción que se da entre sinónimos. ${ }^{10} \mathrm{La}$ otra distinción de razón es la después llamada «de razón racionada» o «de razón razonada», y es la que no depende tan sólo de la mente, sino que está basada en el objeto (cum fundamento in re), y también era llamada «virtual»; se da cuando una cosa se expresa con varios conceptos que - a diferencia de lo que ocurría con la distinción anterior- sí son diversos, por tener diferente definición, de manera que hay fundamento en la realidad para tal distinción, p. ej. el concepto del alma y de sus virtudes o facultades, o entes «animal» $\mathrm{y}$ «racional».

Esta última distinción, de razón razonada, se divide en dos: (i) completa (o mayor): cuando un concepto no incluye al otro, de manera que los contenidos objetivos de esos conceptos pueden realizarse separadamente, p. ej. en el hombre el alma vegetativa, la sensitiva y la intelectiva, porque la primera puede realizarse en la planta, la segunda en el animal y la tercera en los entes superiores o espíritus puros; y (ii) incompleta (o menor): cuando un concepto incluye al otro, de manera que no pueden realizarse separadamente; p. ej. los atributos de Dios.

Tenemos, por lo tanto, esta división de la distinción en la escuela tomista:

\section{Distinción Real}

- mayor (o entitativa, o real simpliciter, o real-real)

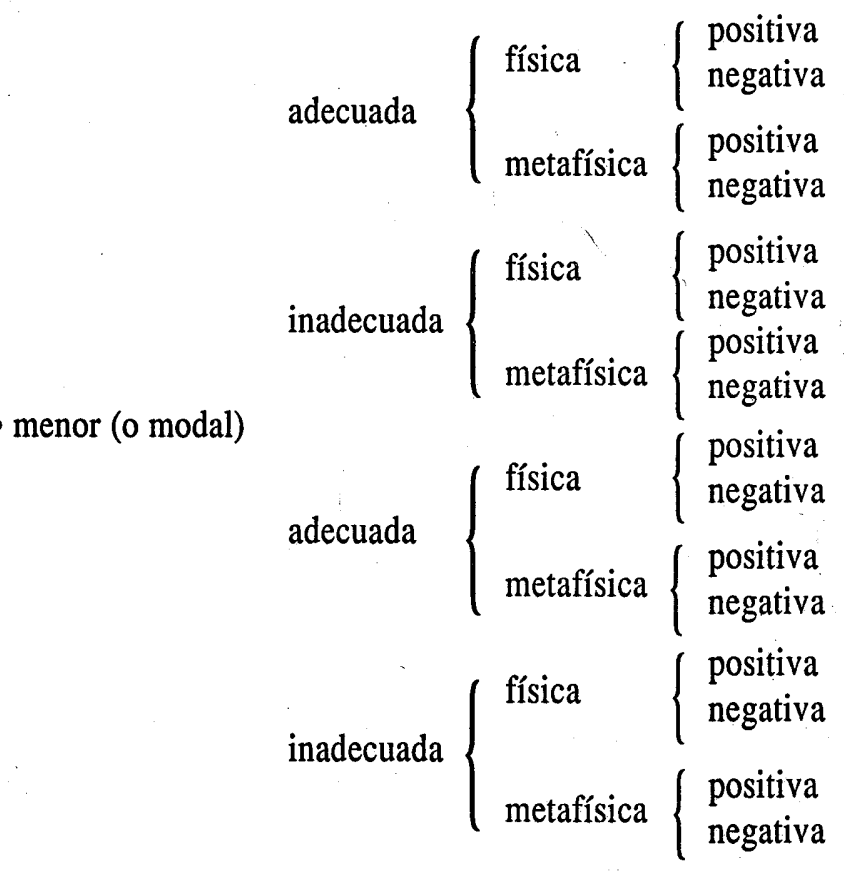

10 Es decir, entre conceptos que tienen sólo nombre diverso pero cuyo contenido ideativo es el mismo. 
Distinción de Razón

- puramente mental

(o de razón raciocinante)

- no puramente mental

(o de razón raciocinante, o con fundamento in re, o virtual)

completa (o mayor, o perfecta, o estrictamente precisiva, o virtual-objetiva)

incompleta (o menor, o imperfecta, o ampliamente precisiva, o virtual formal)

\section{LAS DISTINCIONES EN LA ESCUELA ESCOTISTA: LA DISTINCIÓN FOR- MAL «EX NATURA REI»}

Duns Escoto sigue aceptando las distinciones real y de razón; pero admite otra, que introduce entre ambas, a saber, la formal (formalis ex natura rei). La real es -al igual que para Santo Tomás- la que no depende del intelecto. Se divide en dos: mayor y menor. La mayor, o simpliciter, que es la que media entre entes separables como cosa-y-cosa, tiene cuatro condiciones: «La primera es que sea de algunas cosas en acto, y no sólo en potencia, porque no se distinguen las cosas que están en potencia en la materia, y no simplemente, porque no están en acto. La segunda es que sea de aquellas cosas que tienen un ser formal, no sólo virtual, como los efectos están en la causa virtualmente, no formalmente. La tercera es que sean de aquellas cosas que no tienen un ser confuso como los extremos en el medio y los mezclables en la mezcla, sino de aquellas cosas que tengan un ser distinto con sus propias actualidades. La cuarta, que es la única que completa la distinción perfecta, y la noidentidad, como resulta patente por el Filósofo, donde dice que lo diverso y la distinto son lo mismo»». ${ }^{11} \mathrm{La}$ distinción real menor, o secundum quid, media entre extremos que se relacionan como realidad y realidad (i. e., entre elementos que no son la misma cosa, pero que, unidos, constituyen la misma cosa), como sucede, por ejemplo, con la esencia divina y las relaciones divinas. Y se da cuando sólo se guardan las tres primeras condiciones de la distinción real y falta la cuarta, pues falta la diversidad: «[se da] en cuanto a esas tres condiciones de la distinción propuestas, a saber, no ser tal potencialmente, ni virtualmente, ni confusamente; alli hay verdadera distinción entre tales perfecciones esenciales (divinas), como en algún lugar se encuentra la distinción, porque así verdaderamente en Dios está el intelecto, como si sólo estuviese el intelecto, y de manera semejante la voluntad, y las otras perfecciones de manera simple, y por consiguiente no están allí potencialmente, ni virtualmente, ni confusamente. Pero esas distinciones no bastan para una distinción (simplemente real), porque no introducen no-identidad (o diversidad), y la no-identidad

11 J. Duns Escoto, Reportata Parisiensia, I. d. 33, n. 9; ed. Vives, XXII, pp. 402-403. 
completa la razón de la distinción (de manera simple)». ${ }^{12} Y$ la distinción de razón es aquella en la que el mismo objeto formal se concibe de maneras diversas. ${ }^{13}$

La distinción propiamente escotista es la distinción formal basada en la naturaleza de la cosa (distinctio formalis ex natura rei), es la que se da entre varias formalidades de una cosa. Las formalidades son aspectos reales del ente, sin que la inteligencia pueda fabricarlos, como el alma y sus facultades. La distinción formal se da, pues, entre «dos formalidades de una cosa que en realidad se identifican entre sí, y una de las cuales es, antes de la intervención del intelecto, concebible sin la otra». ${ }^{14}$ Es decir, «la distinción formal es simplemente objetiva, y se da entre dos aspectos, dos realidades, de un ente. Es en lo que se opone a la distinción lógica (o de razón) o subjetiva, que se da entre dos ideas o conceptos. Por ejemplo, el ente contiene en sí las realidades de lo uno, lo verdadero y lo bueno, que son la misma cosa (res) que el ente (ens), y esto por una formalidad quiditativa real; pues de otra manera la metafísica no sería una verdadera ciencia. Si se habla de lo uno, o de lo verdadero, etc., no se mencionan solamente diferentes nombres, sino los conceptos de una misma cosa que conciernen a realidades o formalidades diferentes». ${ }^{15}$ Es una distinción real secundum quid (porque no separa las formalidades como cosa-y-cosa, sino como realidady-realidad, $i$. e., como realidades contenidas unitivamente en una cosa). Así, «el ente contiene unitivamente la razón de lo uno, lo verdadero y lo bueno de los otros, así el alma contiene esas potencias (o sus facultades) unitivamente, aunque formalmente sean distintas». ${ }^{16} \mathrm{Y}$ no corresponde a una composición real estrictamente dicha, pues es virtual y no actual. En efecto, «se requieren dos cosas para una verdadera composición: (a) la separación, por la cual una parte está fuera de la otra, y (b) la mutua perfectibilidad, por la cual una parte se da en orden a la otra a modo de potencia, y la otra en orden a la primera por modo de acto. Pero éstas son extrañas y formalmente distintas. Pues las formalidades, ya que suponen identidad real, aunque se propongan separadas por la abstracción de la mente, son cosas, no la constituyen, y por consiguiente excluyen la razón de parte y de separación. Las formalidades incluyen perfectibilidad sólo en este sentido de que una realidad, por razón de la distinción formal, no incluyendo la formalidad de otra realidad, puede concebirse como perfectible por la formalidad que no tiene; sin embargo, por otra parte, todas las realidades se identifican: 0 (a) porque, como en Dios, por razón del modo intrínseco de la infinitud, una realidad de suyo por la identidad incluye a la otra, o (b) porque, como en las creaturas, las formalidades se ordenan a un tercero (a una cosa) que las incluye unitivamente por la identidad. Las cuales ya se identifican realmente, en cuanto son cosas, excluyen la potencialidad y por ello excluyen la composición real. Si, por lo demás, a alguno le parece irrisorio que a las formalidades se las llame partes, de las que surge la composición, dígase

12 Ibid., I, d. 45, q. 2, n. 9; ed. Vives, XXII, pp. 502-503.

13 Cfr. Idem, Opus Oxoniense, I, d. 8, q. 4, n. 17; ed. Vives, IX, p. 664.

14 Z. Van De Woestine, Scholae Franciscanae adaptatus Cursus Philosophicus, Mechliniae: Typ. S. Francisci, 1933 (2. ${ }^{\mathrm{a}}$ ed.), Ontologia, p. 261.

15 B. de St. Maurice, Jean Duns Scot, París: Gabalda, 1953 (2. ${ }^{a}$ ed.), p. 171

16 J. Duns Escoto, Opus Oxoniense, II, d. II, q. un., n. 17; ed. Vives, XIII, p. 43. 
que las formalidades son partes porque no son toda la perfección de la cosa, y que la composición de formalidades se hace por la mediación del intelecto». ${ }^{17}$

Y es que a cada noción formal le corresponde una cierta entidad. La formalidad es como un medio entre dos contradictorios; no tiene pura existencia mental, sino que atestigua que hay algo entitativo allí, realidades que no se reducen a lo que sólo es de razón. Como toda distinción real, la distinción formal antecede a la intervención del intelecto. Sin embargo, contrariamente a la distinción real simpliciter, que recae sobre cosas no identificadas absolutamente, es una distinción secundum quid, esto es, sólo según un cierto punto de vista. Lo dicho es así, porque ella reside en una cosa real que conserva su identidad absoluta. De esto se sigue que tal distinción es relativa, no es más que una no-identidad formal: «Entiendo - explica Escoto- por no-identidad formal de algunas cosas la que se da cuando una no es la razón formal de la otra, aunque si fuera definida, no pertenecería a la definición de ésta». ${ }^{18}$

La idea de Escoto es que a cada noción que es distinta en su ser objetivo mental o en la mente (in esse objectivo) le corresponde necesariamente una formalidad, realidad, aspecto real, o entidad realmente existente en la cosa, aunque no necesariamente separable. Por eso es una distinción formal basada en la naturaleza de la cosa (distinctio formalis ex natura rei). Tiene una cuasi-existencia o cuasi-entidad en la realidad. Escoto adjudica cierto acto (o ser actual) a las cosas que son en potencia, como la materia, i. e., cambia el sentido a la distinción aristotélica que se recogía en el tomismo. Y por eso admite las formalidades como aspectos reales del ente, como ciertas cosas del ente. $Y$ la distinción que se da entre ellas, cuando se puede pensar con claridad la una sin la otra, es una distinción formal $e x$ natura rei.

Nos quedan, pues, así las distinciones que admite Escoto:

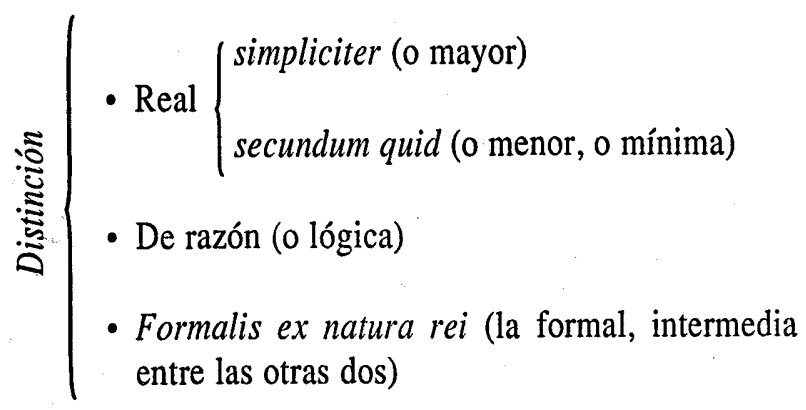

17 Z. Van De Woestine, op. cit., p. 263.

18 J. Duns Escoto, Reportata Parisiensia, I, d. 45, q. 2, n. 9. 


\section{LA REDUCCIÓN DE LAS DISTINCIONES POR OCKHAM EN LA ESCUELA NOMINALISTA}

Ockham se queda, de hecho, con un esquema muy simple de las distinciones. Sólo admite los polos opuestos en sus extremos: la distinción real, entendida como la que se da entre cosa-y-cosa, con separabilidad, y la distinción de razón. ${ }^{19}$ La famosa «navaja de Ockham» rasura todas las distinciones intermedias que pululaban, en el realismo, como pelos en la barba de Platón. Le parecía que era hipostasiar cosas sólo imaginadas. Enemigo sobre todo de Escoto, borra las formalidades y, junto con ellas, la distinción formal y lo mismo hace con las otras que se ponían en el espectro intermedio. Sólo admite la distinción real mayor, física, entre dos cosas que se pueden separar, y además añade la distinción de razón, que ya se subdivide y sólo es la que resulta ser completamente construida por la mente. Así, simplificando las cosas al extremo, en la escuela ockhamista o nominalista, nos queda este esquema de las distinciones:

$$
\text { Distinción }\left\{\begin{array}{l}
\text { Real (sólo la mayor o física) } \\
\text { De razón o lógica (puramente mental) }
\end{array}\right.
$$

\section{LA DISTINCIÓN EN LA ESCUELA SUARISTA: LA DISTINCIÓN REAL}

Suárez acepta la distinción real, como separación entre cosa y cosa; pero da a «cosa» un sentido muy amplio, de modo que puede abarcar tanto a substancias como a accidentes (p. ej. la cantidad). Hay una distinción real positiva, como la que se da entre cosas que son realmente distintas, y otra distinción real negativa, como la que se da entre ente y no ente, o entre entes no absolutamente distintos. Además, como acude a Dios para aquellas cosas que pueden ser separables por su potencia absoluta, resulta que en las distinciones lo más fundamental no es la separabilidad, sino la concebibilidad de los entes distintos. También acepta la distinción de razón, con sus dos clases de razonada (la que tiene fundamento en las cosas) y razonante (la que no lo tiene). Pero además introduce otra distinción intermedia, una que es ex natura rei, pero que no es formal -como la de Escoto (formalis ex natura rei), sino modal, porque «se da siempre entre una cosa y un modo suyo». ${ }^{20}$

Así, Suárez parece revitalizar la distinción formal escotista, sólo que con otro nombre, el de «distinción modal». Pues, en efecto, no es una distinción de razón, sino real, porque

19 G. de Ockham, In I Sententiarum, d. 2, q. 3, B: «Nada se distingue de otro sino como un ente real de otro ente real (sicut ens reale ab ente reali), y toda esa distinción es una distinción real [...]; o se distingue como un ente de razón de otro ente de razón, y toda esa distinción es una distinción de razón [...]; o se distingue como un ente real de un ente de razón, 0 a la inversa, y esa distinción estricta y propiamente no es real ni de razón (nec est realis nec rationis)».

20 F. Suárez, Disputationes Metaphysicae, d. 7, sect. 1, n. 16. 
verdaderamente se da por parte de la cosa y no por una denominación intrínseca que haga el intelecto. Pero no es una distinción real mayor, pues no es una distinción entre cosa-ycosa, con separabilidad, sino que está basada en la naturaleza de la cosa (ex natura rei, llega a llamarla Suárez, con una denominación de neto sabor escotista). Aunque, claro está, el nombre más propio no es «distinción ex natura rei», sino justamente el de «distinción modal». ${ }^{21}$

No se trata, pues, de una distinción entre cosa-y-cosa, o entre ente y ente, sino entre principios o elementos del ente, cuales son los modos. Suárez supone que, «en las cosas creadas, además de sus entidades como substanciales o radicales (por decirlo así), se encuentran algunos modos reales, que también son algo positivo y afectan a las mismas entidades por sí mismos dándoles algo que está fuera de la esencia toda, como individual y existente en la naturaleza de las cosas». ${ }^{22}$ Suárez ve, pues, a los modos no como cosas ni como «entidades substanciales o radicales», sino como «entidades viales», como ciertas realidades 0 aspectos del ente, incluso las llama «entes de ente» (entia entis). Para ejemplificar lo que es un modo, podemos decir que en la substancia hay cantidad; ahora bien, se añade una tercera entidad, que es la unión de ambas; aunque esa unión es una entidad muy débil, es una entidad, es un modo, Es un modo que pertenece a la cantidad, y que se da por virtud de su inherencia a la substancia. En la metafísica de Suárez se da un cambio con respecto a las anteriores: es una metafísica que visualiza más los efectos que las mismas causas, que va más a los acontecimientos que a las formas o esencias, por eso los modos son necesarios, para subrayar las conexiones de las cosas y sus movimientos, tienen una función adverbial en un discurso de flujo, es una metafísica completamente dinámica. Ahora bien, ¿cómo se distingue ese modo de unión o de inherencia con respecto de la cantidad que lo tiene y de la substancia en la que se da? El Eximio responde: «Este modo, tal como ha sido explicado por nosotros, se distingue actualmente, por la naturaleza de la cosa (ex natura rei), de esa cosa de la que es modo, como todos atestiguan; más aún, muchos la llaman distinción real, porque se encuentra en las cosas mismas». ${ }^{23} \mathrm{La}$ distinción modal es, entonces, la que media entre estas determinaciones reales y últimas que son los modos (i. e., entre modo y modo) y entre un modo y la cosa o sujeto al que pertenece.

El signo para reconocer (al menos con probabilidad) una distinción modal es un tipo de separabilidad especial, entre los extremos, a saber, la «separabilidad no mutua», que se da cuando un extremo puede existir separado del otro, pero ese otro sólo puede existir como inherente en el anterior.$^{24}$ Mas, aunque se proclama que éste es el criterio de la distinción modal, criterio todavía muy realista, en la práctica el criterio que propiamente se sigue es la concebibilidad, ${ }^{25}$ el poder ser concebidas en ese estado de separación no mutua las cosas que se distinguen. Dado este requisito de poderse concebir de una manera distinta esos

21 Cfr. Ibid., d. 7, sect. 1, n. 15.

22 Ibid., d. 7, sect. 1 , n. 17

23 Ibid., d. 7, sect. 1, n. 20.

24 Cfr. Ibid., d. 7, sect. 2, n. 6-7.

25 Cfr. Ibid., d. 7, sect. 1, n. 18. 
correlatos (casi diríamos, de una manera clara y distinta), la separabilidad que Suárez atribuye a los elementos de la distinción modal viene a ser la misma separabilidad que concedía Escoto a los extremos de la distinción formal. No parecen muy diferentes (sino marcadamente parecidas) la distinción modal suareciana y la distinción formal escotista.

Queda, así, la distinción en Suárez:

$$
\mid \begin{array}{ll}
\text { Real } & \left\{\begin{array}{l}
\text { positiva } \\
\text { negativa }
\end{array}\right. \\
\text { Ex natura rei o modal }
\end{array}
$$

\section{INFLUENCIAS EN LOS MODERNOS}

Sin duda alguna, el tomismo fue la escuela que menos influyó sobre los modernos. Y, también sin duda alguna, el nominalismo fue el que más influyó en ellos, sobre todo en cuanto actitud. Pero no es menor la influencia del escotismo. Nominalismo y escotismo confluyeron - al igual que, intencionalmente, el tomismo- en la síntesis ecléctica de Suárez. Y a través de él se propagaron en muchos de los modernos. Mas, como el nominalismo excluía la mayor parte de las distinciones, por eso la influencia que conviene señalar es la de Escoto (y su distinción formal ex natura rei) por medio de Suárez (que la reviste del nombre de «distinción modal»). En el ámbito de las distinciones la mayor influencia sobre los modernos fue la de Escoto, por la mediación de Suárez.

En efecto, Escoto no influyó en los modernos por la lectura directa, pero sí a través del ambiente filosófico, tanto nominalista como suareciano (el primero más en Inglaterra y Francia, y el segundo más en España y Alemania). En efecto, Ockham depende mucho de Escoto, tanto positiva como negativamente, y transmite muchas de sus doctrinas, que serán recogidas por sus seguidores nominalistas. Y tanto el escotismo como el nominalismo están presentes en ese filósofo tan influyente que fue Suárez, y que trató de sintetizar a todos (tomistas, escotistas y nominalistas).

La distinción formal de Escoto, que se recoge en la distinción modal de Suárez (al menos en parte), es la que mayormente vemos utilizada por los modernos. Por ejemplo, tanto Escoto como los modernos (especialmente Descartes y Espinoza) utilizan el esse formale y el esse objectivum. Ese mencionado esse objectivum es un preludio del ser intencional fenomenológico, y estos autores - medievales y modernos - quieren hacer 
corresponder lo pensado con lo real, pero partiendo de la pensado. Algo es real y distinto porque así lo concibo. Esto sólo dista un paso de la introducción de las distinciones porque así las capta el entendimiento, y así deben ser en lo real. Es, notoriamente, lo propio de la distinción formal escotista (ex natura rei), que se basa en el esse objectivum y divide las cosas que claramente se piensan como distintas. Si son distintas en el pensamiento, han de serlo en la realidad, corresponden a la cosa, son ex natura rei, y también es ex natura rei - pero basada en el pensamiento o concebibilidad- la distinción modal suareciana. Y todo ello fue bien explotado por los modernos: «Descartes en las Meditations métaphysiques, Spinoza en el Tratado de la reforma del entendimiento y en la Ética, Leibniz en casi la totalidad de sus obras, proponen soluciones a esta dificultad que difieren, ciertamente, por el tema, pero coinciden en la estructura del pensamiento puesto en acción». ${ }^{26} \mathrm{Y}$ tal estructura es la que daban el escotismo y el nominalismo, sintetizados después en Suárez. Es el imperio del esse objectivum escotista y de su famosa distinctio formalis ex natura rei. Y esto se ve claramente en Descartes, en la Lettre à Gibieuf del 19 de enero de 1642: «Creo que todo lo que se encuentra en estas ideas está necesariamente en las cosas». En el terreno de las distinciones, esto corresponde a la mentalidad escotista y suareciana. La intencionalidad se determina no ya por la parte de la cosa, sino por la parte del pensamiento. Es una intencionalidad incluida al lado de la mental, que considera las cosas en cuanto objetos de la mente, esto es, con un esse objectivum mental, y es una intencionalidad (la escotista) que preludia la intencionalidad fenomenológica de hoy en día. ${ }^{27}$

La influencia de Suárez sobre Descartes es harto reconocida, y lo mismo sobre Leibniz. El fundador de la filosofía moderna, Descartes, recoge las distinciones de Suárez, y a través de él pasarán a los más modernos, sobre todo racionalistas. ${ }^{28}$ En los Principes de la philosophie, I, 51-68, se encuentra su mejor exposición. Acepta de la doctrina suareciana la distinción real, la de razón y la modal. La distinción real es, al igual que para Suárez, la separabilidad; incluso toma del filósofo español el que sea al menos por la potencia absoluta de Dios; y, al igual que él, la carga más pesada pende de la concebibilidad. En efecto, Descartes piensa que si algo es separable, tenemos que concebirlo como tal, ya que la realidad no puede sino ser como la pensamos. La distinción de razón por supuesto que también obedece al dictamen del pensamiento. ${ }^{29}$

26 A. de Muralt, «La doctrine médiévale des distinctions et l'intelligibilité de la philosophie moderne (II)», en Revue de Théologie et de la Philosophie, 112 (1980), p. 219, y expresa en pp. 217-218: «Los filósofos modernos no respiran el aire de un escotismo puro, sino que reciben la influencia de un ockhamismo suavizado por Escoto, o de un escotismo mezclado con Ockham, tal como lo realiza por ejemplo ese poderoso sincretismo de intención aristotélica que es la obra, universalmentre extendida en los siglos XVI y XVII, del jesuita Suárez».

27 Cfr. Idem, «Deux aspects contemporains de la philosophie de l'esse obiectivum», en Freiburger Zeitschrift für Philosophie und Theologie, 34 (1987), pp. 161-168.

28 Cfr. E. Gilson, Index scolastico-cartésien, París: Vrin, 1979, p. 87.

29 Cfr. J. Zubimendi Martínez, «La teoría de las distinciones de Suárez y Descartes», en Pensamiento, 40 (1984), p. 188 ss. 
En cuanto a la distinción modal, la propiamente suareciana, fue la que más impacto tuvo en el sistema cartesiano, ya que adopta la teoría de los modos de Suárez, ${ }^{30}$ que después pasarán singularmente a un pensador tan atento a la modalidad como Espinoza. Algo también notable en lo que Descartes coincide con Suárez es en considerar que la distinción formal de Escoto viene a ser lo mismo que la distinción modal. Hablando de Catherus, Descartes dice: «por lo que mira a la distinción formal que ese muy docto teólogo dice haber tomado de Escoto, respondo brevemente que, no difiere de la modal, y que no se extiende más que a los seres incompletos, los cuales cuidadosamente he distinguido de los que son completos». ${ }^{31}$ Con todo, Descartes cambia la noción suareciana de modo y le da su sello específico: «¿Cómo se pasa de este concepto suareciano de modo al específicamente cartesiano? La respuesta es bien sencilla: por la negación de que haya accidentes reales. Todo lo que es real y separable natural o sobrenaturalmente es según Descartes substancia. Lo no separable será, pues, modo». ${ }^{32}$

Incluso lo que Descartes llama «atributos» o «cualidades» no son otra cosa sino modos; son atributos que se distinguen de la substancia con distinción real. Y en cada substancia hay un atributo principal, que constituye su naturaleza o esencia, y de él dependen los demás atributos; éste sólo se distingue de la substancia con distinción de razón. Pero «la falta de aclaración fundamental en el estatuto del atributo esencial provocará incongruencias categoriales que Espinoza explotará. Una mente se puede distinguir realmente de otra, porque puede existir sin ella. La esencia de ambas es el pensamiento, que en cuanto se considere diversificado en una y en otra, no tendrá otro rango que modal y, por tanto, no parece que se pueda establecer entre ambas mentes otra distinción que la modal. Esto se ve agravado si consideramos el caso de la extensión, pues, aunque no se suela decir que el pensamiento incluye todos los pensamientos, es fácil decir que la extensión contiene a todas las extensiones como sus partes o modificaciones. Tengamos en cuanta que la substancia se puede pensar sin este o aquel modo, pero no sin modos en absoluto, por lo que aunque Descartes mantenga que hay distinción real entre mentes numéricamente distintas, la definición de las mentes a través del atributo, que en cuanto se considere numéricamente diversificado caerá a nivel modal, generará las incongruencias que Espinoza desarrollará en el principio de la Ethica. Descartes ha olvidado el ser mental en favor de sus acciones, manteniendo la diferencia entre ambos impensadamente. El trabajo de Espinoza consistirá en suprimirla, haciendo que la mente sea sus ideas y que el cuerpo sea sus acciones». ${ }^{33} \mathrm{Con}$ eso la teoría de las distinciones pierde su carácter escolástico y se toca de manera plena la presencia de la modernidad, casi como será y nos ha llegado hasta ahora.

30 Sobre la recepción cartesiana de la teoría suareciana de los modos, cfr. E. Gilson, Études sur le rôle de la pensée médiévale dans la formation du système cartésien, París: Vrin, 1927, pp. 141 ss. Allí se estudia el paso de la física escolástica de las formas substanciales a la física escolástica de los modos extensionales, o cuantitativa.

31 R. Descartes, Réponses aux premières objections, en Oevres, ed. Adam-Tannery, IX, 94.

32 J. Zubimendi Martínez, art. cit., p. 196.

33 Ibid., pp. 201-202. 